to himself when spoken to. The pupils were irregular, the right being slightly the larger; both were active. There was divergent strabismus of the right eye, and the mouth was slighity drawn to the left. There was partial paralysis of the right side. He used the left hand more in feeding himself, but could hold the spoon in the right, clutching the handle in the palm with the whole hand-the fine movements for the fingers being apparently lost-and thus he put the spoon to his mouth. He was weak on his legs, and in walking lifted the left leg with a slight jerk, somewhat after the manner of a stringhalt horse. When left standing up unsupported, he leant or walked backwards, vertical equilibrium being apparently interfered with. The sole of the left foot was sensitive to tickling.

October 15 th. - The movements of the limbs varied very much, while equilibrium (vertical) was disturbed; the gait to-day was more like that of a drunken man. He was sweating profusely this morning.

October 23rd.- He could not walk unsupported; when he tried to get out of a chair, he fell backwards; and if walking by support, leant backwards. He dragged the right leg, and put out the left without proper control. He did not use the right hand in feeding himself, but if allowed groped about on the plate with the left, even after he had eaten all off it. He soiled his clothing, and crammed the food ravenously into his mouth, unless fed. His mental condition was that of organic dementia.

October 29th. - He had become much feebler, and had lost almost all power of motion in the lower limbs; apparently, his sight was almost entirely gone. Sensation, as tested by tickling the soles of the feet and calves of the legs, was present, but apparently somewhat stronger on the left side. He was in a stupid, drowsy, and sleepy condition. Continuing in this drowsy and heavy state, he went off rapidly and unexpectedly, dying at 7.30 P.M.

Post mortem Examination. - The scalp was tough, and highly vascular; the calvarium oblong and fairly symmetrical. On its internal aspect, in the grooves for the meningeal vessels, and on the parts adjacent to these, was seen a blood-stained exudation; this was.also seen

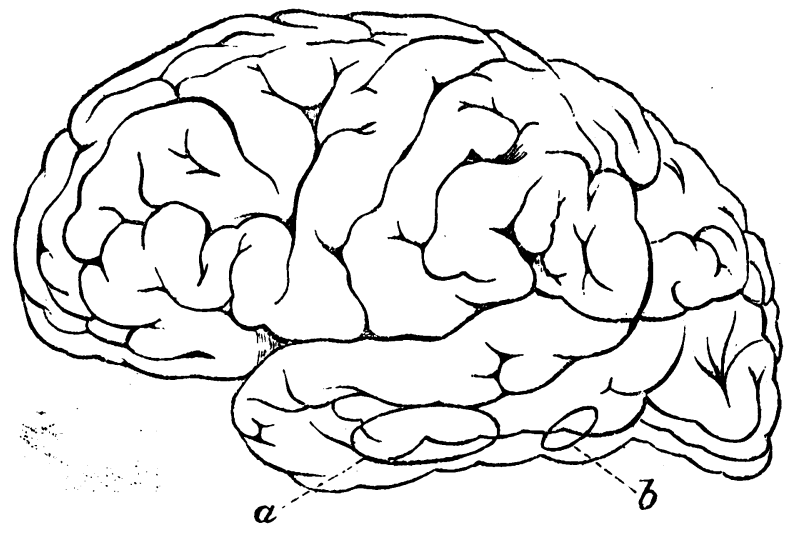

a. Tumour. 6. Opening into disorganised interior.

on the corresponding aspects of the dura mater. In the removal of the encephalon, very little fluid escaped. The brain weighed sixty ounces (avoirdupois); "it was plump, and so completely filled up its cavity that the convolutions were quite smooth and the sulci almost obliterated, or marked by faint lines. There were no large veins ramifying on its surface ; nor was there any adhesion of the dura mater along the edges of the longitudinal sinus, or elsewhere. The sinuses at the base of the skull were engorged with blood. There was no opacity of the arachnoid or of the pia mater. On being placed on its under surface, $a$, distinct bulging, with a breach of continuity of the grey matter of the second and part of the third left temporo-sphenoidal convolutior, came into view. On examination, this was found to be due to a tumour (or tumours) of oblong form, occupying a large portion of this lobe. The surrounding structure was softened and broken down, the greater part of the interior of the lobe being thus destroyed; while posterior to and below the breach already described, an opening was seen communicating with the disorganised interior. The white substance was unusually pale and doughy; otherwise, the organ, to the naked eye, appeared healthy.

My friend, Dr. Bevan Lewis, of the West Riding Asylum, has kindly sent me the following note as to the nature, measurements, and microscopic appearances of the tumour. "It was purely an encysted hamorrhage, the whole mass measuring two inches and a quarter in its greater, by one inch and a quarter in its shorter, diameter. It had a tough fibrinous boundary, and centrally was a mass of blood.corpuscles and fibrine, which broke down readily in attempting to make sections of the hardened preparation, leaving a fine fibrinous reticulum."

\section{FOUR CASES OF CYSTINE CALCULI.}

By F. A. SOUTHAM, M.B., F.R.C.S.,

Cystine calculi are of such rare occurrence, when compared with other varieties of urinary concretions, that the following particulars of four cases which occurred in the practice of my father (the late Mr. George Southam) are, I think, worthy of record.

CASE I.-Margaret P., married, aged 33, residing at Ashton, was admitted into the Manchester Royal Infirmary on September 14th, 1868, suffering from well-marked symptoms of stone. On October r6th, the operation of lithotrity was performed, the urethra being rapidly dilated and the calculus removed entire. The operation was, as is usually the case, followed by incontinence of urine; but this soon disappeared, and she was discharged from the hospital on November 9th with perfect command over her bladder.

The calculus was pure cystine; weight 180 grains; shape oval; size an inch and $a$ half by an inch. The surface was slightly tuberculated, glistening, and of a dirty brownish-yellow colour. Some difficulty was experienced in making a section of the calculus, owing to its soft consistence and tendency to crumble down; but it shows a whitish waxy surface. The central portion has a chalky appearance, forming an indistinct nucleus, and is of a whiter colour than the more external portion, which shows a somewhat radiated arrangement.

Having ascertained that the patient was still living at Ashton, I visited her in June 1877 , with the view of making some inquiries into her family history and present condition. She is one of a family of nine, who are all alive and healthy. No member of her family, either on her father's or mother's side, has, as far as she is aware, ever suffered from stone or any symptoms referable to the bladder. She was always strong and enjoyed good health until the age of thirty-two, when she began to be troubled with symptoms of stone, which were very severe for some months preceding the operation. She has had four children, all now alive; of these, three were born before the operation; the youngest, a girl, was born four years subsequently to the operation, and is now consequently five years of age. She is an anæmic strumous-looking child, with enlarged cervical glands, and has always had very delicate health. The patient herself is a slightly built woman, of delicate appearance and sallow complexion. She has never been very strong since the operation, though able to get about and attend to a small shop of which she has the charge. She has never noticed any gravel in her urine, or suffered from attacks of renal colic, since the operation. So long as she keeps tolerably quiet, she is able to retain her urine perfectly; but, if she undertakes any hard work or takes much exercise, she is troubled with incontinence.

Being anxious to ascertain whether the condition of cystinuria, which must have been present eight years ago, still existed, I obtained some of her urine for examination, and found that it presented the following characteristics. It was of specific gravity 1017 , turbid when voided; had a peculiar aromatic odour and a pale amber colour; was faintly acid. On standing, it very quickly deposited a copious whitish sediment, the supernatant fluid remaining very turbid. Examination of the deposit under the microscope showed very numerous transparent hexagonal crystals of cystine, the field being crowded with them. Mingled with these was a slight quantity of amorphous urates. On the addition of hydrochloric acid or ammonia, the crystals at once disappeared. On boiling a little of the urine with potash and leadacetate, a dark-coloured precipitate of lead-sulphide appeared. After standing twenty-four hours, the urine became alkaline, with a strong and disagreeable odour. After forty-eight hours, the crystals of cystine had entirely disappeared, numerous crystals of triple phosphate being present.

An examination of the urine of the daughter, aged 5 years, gave the following results: Specific gravity 1010; neutral; very pale strawcolour ; quite free from any odour; quite clear; no deposit on stand. ing. Microscopic examination did not show the presence of any crystals, nor was the addition of acetic acid or solution of carbonate of ammonia followed by the precipitation of any crystals of cystine.

CASR II.-Alice W., aged 4, residing at Bury, came under my father's care in 1869 , suffering from symptoms of calculus resicze. The urethra having been dilated, lithotrity was performed, and the fragments of the calculus subsequently escaped in minute particles, the 
Inrgest of which are about the size of a pea; they have a yellowish waxy glistening appearance, and consist of pure cystine. The weight of the fragments is eighty-eight grains.

CASE III.- James B., aged IO, residing at Oldham, was admitted into the Manchester Royal Infirmary in March 1870, with symptoms of stone. Lateral lithotomy was performed on May 13 th, and he was discharged cured on June 1oth. The calculus, which consists of pure cystine, was fractured into three portions during extraction. When put together, the fragments form an oval calculus, about an inch by an inch and a quarter in size. In appearance, it is very similar to specimen No. 1 , showing a raciating arrangement, but no indication of any central nucleus. The weight is one hundred and fifty grains.

CaSE IV.-John M., aged 57, residing at Staleybridge, was admitted into the hospital in June 1874. Lithotrity was performed on June $14^{\text {th }}$ and $24^{\text {th }}$, and the patient eventually succumbed to a severe attack of bronchitis. The total weight of the fragments of the calculus, which consisted entirely of cystine, was one hundred and ninety-five grains. Full particulars of this case were published in the BRITISH Medical JouRnal, December 23rd, 1876.

REMARKS. - The preceding four cases of cystine calculi occur in a collection of fifty.five specimens which were all removed by my father, and are now in my possession. This is an unusually large proportion, when we consider the comparative rarity of this variety of urinary concretion; for in the Museum of the Royal College of Surgeons, out of a total of about one thousand calculi, there are only five specimens of cystine; and in the Museum of Guy's Hospital, out of about five hundred and twenty calculi, there are only six specimens of this variety of calculus. I regret that I have been unable to find any further record of Cases II and III, and all attempts to trace the patients have proved unsuccessful.

It is, perhaps, worthy of remark, that three out of the four cases came from the same locality; that two of the cases occurred in females; and that, in the two cases in which $I$ was able to inquire into the family history, there was no evidence of calculus having been ever present in any other members of the same family.

\section{IDIOPATHIC MYDRIASIS, TREATED WITH ESERINE : GOOD RECOVERY.}

BY SAMUEL BENTON, L.R.C.P., M.R.C.S.,

Iate Assistant Medical Officer to the Central London Sick Asylum Infirmary, Highgate.

NuRse B., aged 26, consulted me on May 14th, 1877 . She was suffer. ing from great pain in the left eye, occasioned (as was at once apparent) by dilatation of the pupil ; the conjunctiva was also a little inflamed, but this soon subsided. The day before I saw her, she had experienced some little pain in the eye after reading, and they occasionally watered. The patient steadfastly denied putting atropine into or otherwise tampering with her eye.

History. - She was not the subject of hysteria; did not sufier from worms, and had not had syphilis or rheumatism ; but was a healthy young woman. The only illness she rememhered having was typhoid fever in 1872 ; a severe attack, but from which she made a good recovery.

There was no strabismus, and both eyes were of normal tension. The left pupil was very much dilated, and would not yield to strong light. It was interesting to notice the sympathetic movenents of the iris in the right eye, when the hand was placed over its fellow. The disc and fundus of the left eye were perfectly normal.

I ordered an aperient, and a mixture containing two grains of quinine, twice a- day; chloral and camphor liniment to the brow; also fwo Calabar bean discs to be introduced into the eye.

May 15th. The Calabar bean discs had failed to contract the pupil. There was acute pain in the left temple and down that side of the face.

May 1 th. There was no abatement of the symptoms. The quinine was discontinued ; and fifteen minims of tincture of perchloride of iron, and five minims of liquor strychnix, were ordered to be taken three times a day.

May rgth. The pupil was still largely dilated. The pain was severe across the forehead. There was some photophobia ; no strabismus. Distant objects could alone be discerned with the left eye.

On May 2oth, six days after the commencement of the illness, there was no alteration in the symptoms. Mr. Bowater J. Vernon kindly saw the patient with me : he confirmed the diagnosis, gave a favourable prognosis, and suggested the advisability of trying alkalies. She was, therefore, ordered to have the following draught three times a day: R Potassæ bicarbonatis gra. xv; potassii iodidi gra. $x$; aquæ $q . s$. ad 3 i. A blister was ordered to be applied to the left temple.
May 21st. There was no alteration. The pain was very severe all round the left brow, and was felt down the lett side of the nose. The patient had no sleep.

May 22nd. Three Calabar bean discs were introduced into the eye, with a negative result.

May 23rd. The pain in the eye continued. She slept badly. B Liquoris strychnix $\mathrm{m} v$; tincturx aconiti $\mathrm{m} v$; aquæ ad $\mathfrak{y i}^{\text {. Fiat }}$ haustus ter die sumendus.

May 24th. One drop of a solution of extract of Calabar bean (two grains to one ounce) was introduced into the left eye.

May 25th. The iris had not yet acted, and the pain was so severe that she was unable to sleep at night.

May 27th. She was sent out of doors for a short walk, in the hope that the sun might stimulate the iris to act.

May 28th. The pupil was still dilated, accompanied with pain in the left temple so excessive that she had not slept for several nights.

May 29th. A subcutaneous injection of one-sixth of a grain of morphia was made last evening, which gave a better night's rest.

May 3oth. The morphia injection was repeated. She was ordered three leeches; two applied to the outer, and one to the inner canthus of the left eye.

May 3 Ist. There was no improvement. On examination with the ophthalmoscope, the left pupil was found immovable. There was no diminution of the anterior chamber. The disc, at its upper part, was a little pigmented; but this condition existed in both eyes. There was no cupping of the discs, and the fundus appeared quite healthy.

June ist. One drop of liquor atropize was put into the eye, but it took no effect upon the iris.

June 5th. Another leech was applied.

June 6th. There was no alteration in the symptoms. The left pupil was still dilated, and there was a stabbing pain in this eye at times, in. creased if anything at night. The tension of the eye remains normal.

June 7 th. One drop solution of one grain of eserine in a drachm of water was dropped into the left eye, and, half an hour afterwards, the pupil contracted.

June 8 th. She had less pain. The left pupil was a little more dilated than the right.

June 9th. Another drop of the eserine solution was introduced into the left eye, and the patient sent into the country. In a week's time, she was quite well; both pupils acting, and there being no squint and no impairment of vision.

REMARKS. - I am aware that cases of mydriasis, often due to various remote constitutional causes, are not uncommon occurrences in weakly patients, the exciting cause being a dranght or sudden gust of wind as that met with in looking out of a railway-carriage ; but it is seldom one meets with such an obstinate case as the one I have referred to, extending as it did over three wceks. The above case seems to be occasioned by a freak of the radiating fibres of the third nerve, with some irritation of the sympathetic, probably due to worry and overwork; and is interesting in showing how eserine will sometimes restore power in the iris, when Calabar bean, atropine, and opium have no effect upon it. The impairment of vision was due solely to the dilated pupil ; there was no loss of accommodation, as the patient could see to reacl quite plainly through a pinhole aperture in a piece of card held close to the eye. I may mention that the eserine used came from Darmstadt, as the sulphate of eserine at present sold in chemists' shops often fails to have effect upon the iris.

\section{A CASE OF PARALYSIS OF THE ABDUCTORS OF THE VOCAL CORDS.}

\section{BY SOLOMON CHARLES SMITH, F.R.C.P.Ed., Surgeon to the Halifax Infirmary.}

A san came under my observation some time ago, whose symptoms appeared to be due to paralysis of the abductors of the vocal cords, and, as Dr. Semon's paper before the Clinical Society, and the discussion which followed it, indicate the rarity of the affection, it is probably worth recording.

I first saw the patient on May 16th, I876, when I found him sitting up in bed, firmly grasping the bedclothes, breathing with the utmost difficulty, every inspiration being accompanied by a loud whistling croupy sound, and being frequently interrupted altogether, so that it was both noisy and jerky, giving the idea that it was interfered with by some loose valvular Hap hanging over the glottis. The expiration was, however, perfectly easy, and the voice was hardly affected. When watching the distressed, noisy, laborious manner in which he drew 